\title{
Monosporium apiospermum meningoencephalitis: a clinico-pathological case
}

\author{
I Durieu, M Parent, F Ajana, P Gosset, D Smadja, X Leclerc, Y Fournier, D Leys
}

\begin{abstract}
Twenty nine cases of central nervous system infection due to Monosporium apiospermum have been reported. Six of them occurred after an aspiration pneumonia following a near drowning. The case of a 53 year old man is reported: $M$ apiospermum was isolated from cerebrospinal fluid, sputum, urine and from the mud of the ditch where the patient had fallen. Though the treatment used recommended doses of intravenous, intrathecal and intraventricular amphotericin $B$, it was ineffective and the patient died 97 days after the near drowning. Necropsy showed that the ventricular infectious process was more prominent distant from the antifungal ventricular source.
\end{abstract}

Monosporium apiospermum is the asexual form of Pseudallescheria boydii. This ubiquitous fungus commonly found in soil, rivers, and polluted water is a well known cause of cutaneous, pulmonary and osteo-articular infections. However, 29 cases of CNS infections have been previously reported. Aspiration pneumonia following a near drowning is, after immunodepression, the main predisposing factor for $M$. apiospermum infections of the CNS. ${ }^{1-6}$ The prognosis depends on the severity when the treatment is started, ${ }^{4}$ and is usually bad because of severe difficulties encountered in making an ante mortem diagnosis. ${ }^{7}$ We report the following case because of the rare occurrence of $M$. apiospermum infections of the CNS, the difficulties in making the diagnosis and the fatal evolution despite an adequate treatment.

Case report

A 53 year old previously healthy market gardener, developed an aspiration pneumonia after he was found lying face down in a ditch after a car crash. He had no head trauma. Clinical and radiological improvement of the pneumonia was obtained within 15 days under netilmicin ( $400 \mathrm{mg}$ IM daily), cefotaxime ( $3 \mathrm{~g}$ IV daily), and methyl-prednisolone (40 $\mathrm{mg}$ IV daily) for seven days, then under pefloxacin (800 mg IV daily), erythromycine (4 g IV daily) and chloramphenicol (2 g IV daily) for 24 days. On the 30 th day after the accident his temperature was $38.5^{\circ} \mathrm{C}$ and antibiotics were withdrawn to perform new bacteriological samples. Twenty four hours later confusion, frontal headache and mild neck stiffness appeared while the remaining neurological examination was normal. CSF was clear with normal pressure and contained 280 leukocytes $/ \mathrm{mm} 3$ ( $76 \%$ polymorphonuclear), 180 $\mathrm{mg} / \mathrm{dl}$ proteins and $29 \mathrm{mg} / \mathrm{dl}$ glucose; CSF cultures were sterile. Brain CT scan revealed a left cerebellar lesion suggesting an abscess. Staphylococcus dermatitis was isolated from the blood cultures and catheter, and the patient was treated with rifampicin $(1800 \mathrm{mg}$ IV daily), vancomycin (1500 mg IV daily) and ornidazole (1 g IV daily). Hydrocortisone (50 $\mathrm{mg}$ ) and chloramphenicol (50 $\mathrm{mg})$ were administered intrathecally every three days. The temperature fell to $37^{\circ} \mathrm{C}$ within three days but the patient remained confused. Thrombocytopaenia $(75,000$ platelets $/ \mathrm{mm} 3)$ was seen between day $47-50$, and on the fifty first day, transient macular rashes appeared on

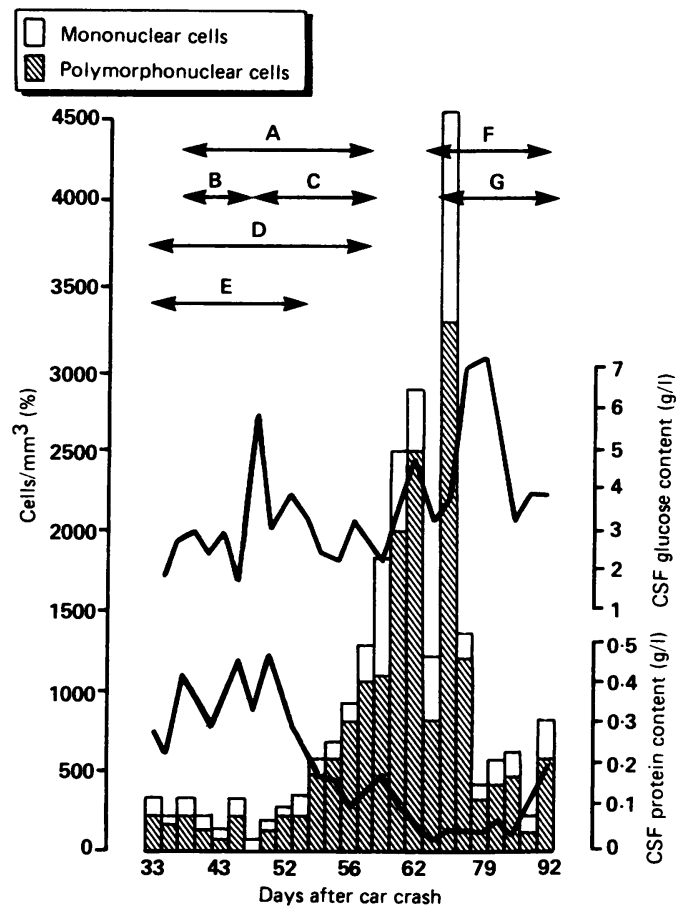

Figure 1 CSF evolution under treatment from the 32nd to the 93rd day after car crash. (a) intrathecal chloramphenicol ( $50 \mathrm{mg}$ every three then every two

days); (b) intrathecal hydrocortisone $(50 \mathrm{mg}$ every three days); (c) intrathecal hydrocortisone (100 mg every two days); (d) intravenous therapy with rifampicine (1800 $\mathrm{mg} / \mathrm{d})$ and vancomycine (1500 $\mathrm{mg} / \mathrm{d}) ;(e)$ intravenous ornidazole $(1 \mathrm{~g} / \mathrm{d}) ;(f)$ intraventricular amphotericine $B$ ( $0.1 \mathrm{mg}$ to $0.9 \mathrm{mg}$ every four days) + intravenous amphotericine $B$ (from 5 to $55 \mathrm{mg}$ every two days) + intravenous flucytosine ( $5 \mathrm{~g} / \mathrm{d}) ;(\mathrm{g})$ intrathecal amphotericine $B$ (from $0.1 \mathrm{mg}$ to $0.6 \mathrm{mg}$ every two days). 


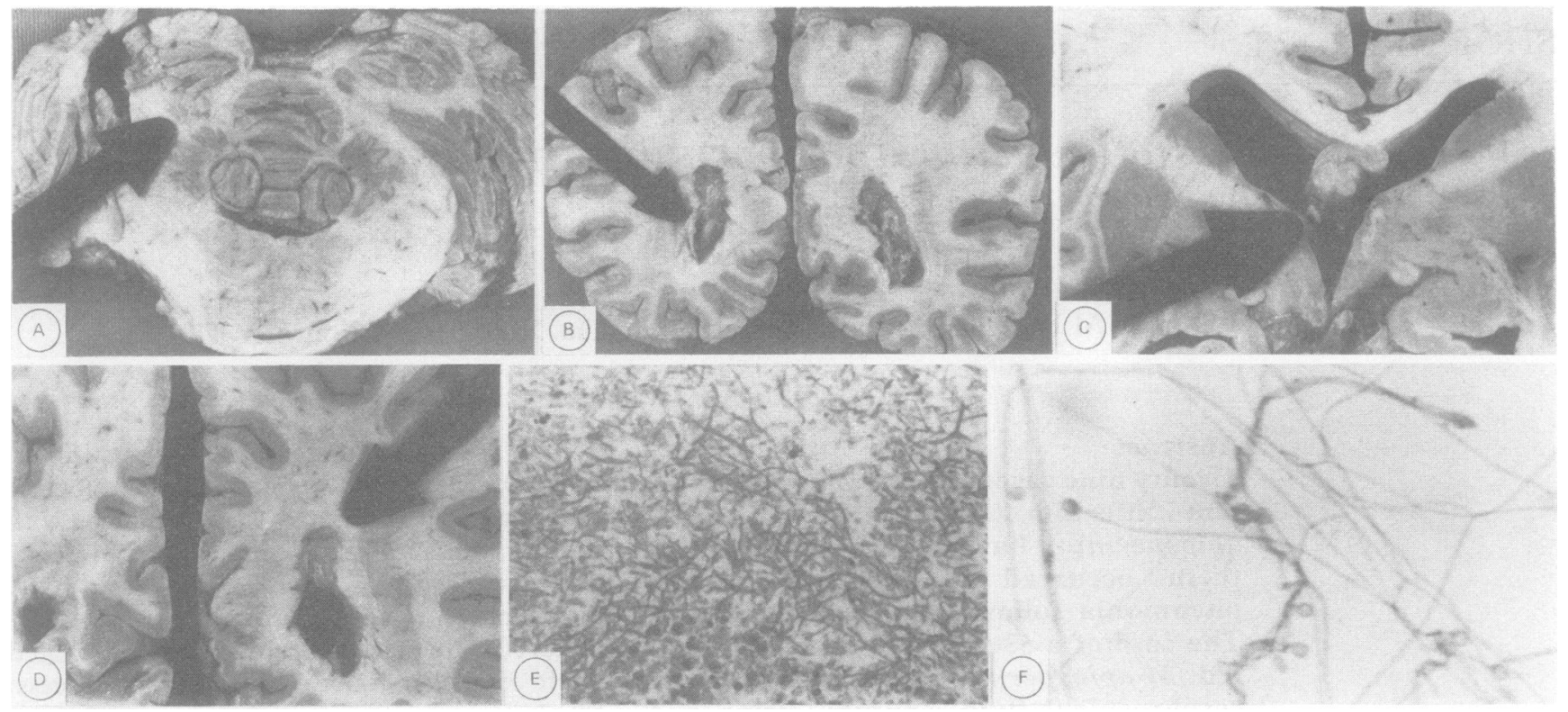

Figure 2 Pathological findings; (a) cerebellar nodule (arrow); (b) intraventricular purulent material (arrow); (c) intraluminal isolated spicule (arrow); (d) periventricular nodule (arrow); (e) fungal filaments isolated from purulent material in occipital horns (PAS strain $\times 400$ ); ( $)$ culture of Monosporium apiospermum in Sabouraud medium showing single conidia arise on the tip of conidopheres (lactophenol cotton blue preparation $\times 400$ ).

the trunk. CT scan revealed the same cerebellar lesion and multiple nodules appeared in the periventricular white matter together with an enhanced ependymal reaction within the occipital horns. CSF follow up under antibiotics is reported in figure 1. On day 55 , his confusion deteriorated but the temperature was $37^{\circ} \mathrm{C}$ and neurological examination remained otherwise normal. After a second break from medication, CSF contained 2560 leukocytes $/ \mathrm{mm} 3$ ( $80 \%$ polymorphonuclear), $3540 \mathrm{mg} / \mathrm{dl}$ proteins and 10 $\mathrm{mg} / \mathrm{dl}$ glucose. $M$ apiospermum was isolated from the CSF, sputum and urines, but blood cultures remained sterile. $M$ apiospermum was also isolated from a sample of the mud from the ditch where the patient had fallen two months earlier (Sabouraud's culture medium, lactophenol cotton blue). The isolated strain was susceptible to amphotericin B, flucytosine, miconazole, ketoconazole and itraconazole. Treatment started with increasing doses of amphotericine $B$, beginning with $5 \mathrm{mg}$ the first day up to $55 \mathrm{mg}$. Amphotericine was administered each two days (one day with and one day without), with effective blood levels. The patient also received intrathecal and intraventricular amphotericine B (respectively from $0.1 \mathrm{mg}$ to $0.6 \mathrm{mg}$ and from $0.1 \mathrm{mg}$ to $0.9 \mathrm{mg}$ per injection), and intravenous flucytosine ( $5 \mathrm{~g}$ daily). CSF cultures were negative within 10 days, whereas urine and sputum cultures were still positive. The level of consciousness rapidly worsened, and hydrocephalus appeared. On day 94, the acute hydrocephalus was reduced by a ventricular puncture through the subcutaneous device. Three days later, the patient suddenly died. $\mathrm{He}$ had received total intravenous doses of $853 \mathrm{mg}$ amphotericin B, $150 \mathrm{~g}$ flucytosine, intraventricular doses of $6.6 \mathrm{mg}$ amphotericin $\mathrm{B}$, and an intrathecal dose of $2.7 \mathrm{mg}$ amphotericine $B$.

General necropsy revealed a bilateral bronchopneumonia with rare mycelial filaments in the bronchiolar lumen. The other organs had no fungal elements: kidneys, spleen, myocardium and endocardium were normal. We found also a chronic aspecific pachyvaginalitis. The immediate cause of death was not clearly established.

The fixed brain weighed $1300 \mathrm{~g}$. Necropsy (fig 2 ) revealed a generalised cerebral oedema, with right temporal herniation. The thickened leptomeninges had an inflammatory aspect, but they were free of any filament. The left cerebellar lesion $(8 \mathrm{~mm})$ was at a chronic stage, without any fungal filaments. An easily separated material filled the occipital ventricular horns, including the purulent choroid plexus. A well circumscribed lesion overlapped the roof of the right ventricular horn to lie in the periventricular white matter. In the anterior part of the lateral ventricles, this material was less invasive and was developed from the roof of the ventricle. Microscopic examination of the yellowish material showed an acute suppurative necrosis bordered by granulomatous reaction with new vessels containing mycelial filaments: these were septate hyphae, with little branching, sometimes rigid or regularly dilated, but no sporulation.

\section{Discussion}

Isolation of $M$ apiospermum from the ditch where the patient had fallen and from the sputum made the lungs the probable route of entry. The causative agent was not detected in blood cultures but blood dissemination was probable since we found $M$ apiospermum in the urine and CSF cultures. Initially clinical improvement with antibiotics was misleading for the diagnosis. However, the improvement was too slow, and the appearance of CT abnormalities, within the white matter, made us consider a fungal infection in spite of negative results of CSF cultures performed 15 
days before the diagnosis. Unfortunately, intrathecal hydrocortisone probably made the fungal dissemination easier. Systemic steroids, initially given for the pneumonia, probably favoured the pulmonary dissemination of $M$ apiospermum. The fungal septicaemia probably occurred when cutaneous manifestations and transient thrombopaenia appeared as reported in a previous case. ${ }^{2}$ Local and systemic amphotericin $B$ made CSF cultures negative within 10 days. Necropsy examination revealed that CT scan signs of ventriculitis were due to the infectious process, and not to the toxicity of intraventricular amphotericin B. There was more purulent material at a distance from the local source of antifungal therapy, in the right frontal horn. This posterior predominance of fungal ventriculitis far from the catheter emergence, leads us to believe that our local therapy was partially effective but was not sufficient to stop the infectious process spreading posteriorly. However, posterior areas were initially more severely involved than anterior areas and the position of the patient in his bed might have favoured the posterior predominance of infection too. Cerebral localisation makes fungi particularly inaccessible to treatment, ${ }^{8-10}$ and total recovery is unusual. ${ }^{10}$

We thank Miss Y Kucharski for secretarial assistance.

1 Dubeau F, Roy LE, Allard J, et al. Brain abscess due to Petriellicium boydii. Can J Neurol Sci 1984;11:395-8.

2 Gari M, Fruit J, Rousseaux P, et al. Scedosporium (Monosporium apiospermum: multiple brain abscesses. Sabouraudia 1985;23:371-6.

3 Travis LB, Roberts GD, Wilson WR. Clinical significance of Pseudallescheria boydii: A review of 10 years' experience. Mayo Clin Proc 1985;60:531-7.

4 Fischer JF, Shadomy S, Teabeaut JR, et al. Near-Drowning complicated by brain abscess due to Petriellidium boydii. Arch Neurol 1982;39:511-3.

5 Meadow WL, Tipple MA, Rippon JW. Endophtalmitis caused by Petriellidium boydii. Am I Dis Child 1981 135:378-80.

6 Kershaw P, Freeman R, Templeton D et al. Pseudollescheria boydii infection of the central nervous system. Arch Neurol 990;47:468-72.

7 Guyotat D, Piens MA, Bouvier R, Fiere D. A case of disseminated Scedosporium apiospermum infection after bone marrow transplantation. Mykosen 1987;30:151-4.

8 Schwartz DA, Amenta PS, Finkelstein SD. Cerebra Pseudallescheria boydii infection: unique occurrence of fungus ball formation in the brain. Clin Neurol Neurosurg 1989;91:79-84.

9 Lavarde V, Farge D, Sabine V, Dupont B, Mourier KL. Brain abscess due to Scedosporium apiospermum in a renal transplant patient. Journal of Acquired Immune Deficiency Syndromes 1988;1:201-2.

10 Perez RE, Smith M, McClendon J, Kim J, Eugenio N. Pseudallescheria boydii brain abscess. Complication of an intravenous catheter. Am J Med 1988;84:359-62. 